\title{
Quantum interference effects
}

\section{in $N^{*}$ electroproduction and propagation in nuclei}

\author{
A. Bianconi, S. Boffi and D.E. Kharzeev 由 \\ Dipartimento di Fisica Nucleare e Teorica, Università di Pavia, and \\ Istituto Nazionale di Fisica Nucleare, Sezione di Pavia, Pavia, Italy
}

\begin{abstract}
We discuss the unexpected enhancement of the $N^{*}$ electroproduction on nuclei recently observed in the $\left(\gamma^{*}, p \pi^{-}\right)$measurement. A mechanism which is able to explain this result is proposed. To clarify the situation, we suggest to perform a new kind of experiment within well specified kinematic conditions.
\end{abstract}

Recently a measurement has been reported [1] of the $\left(\gamma^{*}, \pi^{-} p\right)$ reaction in the second resonance region $(W \approx 1530 \mathrm{MeV})$ at different values of the invariant mass $W$ and of the missing momentum $p_{m}$. This provides valuable information on $N^{*}$ electroproduction and propagation in nuclei. In the same work the data have been compared with theoretical expectations based on a

\footnotetext{
${ }^{1}$ On leave from Moscow University, Moscow, Russian Federation
} 
$\operatorname{DWBA}\left(\gamma^{*}, \pi^{-} p\right)$ calculation. The new result is that, for $W \approx 1530 \mathrm{MeV}$, at high $p_{m}(222 \mathrm{MeV})$ the data to DWBA ratio is $1.9 \pm 0.3$ (while at $p_{m}$ $=56 \mathrm{MeV}$ this ratio is $\approx 1$ ). After discussing the possible causes of this mismatch, the authors of Ref. [1] suggest that it could be due to Final State Interactions (FSI) between $N^{*}$ and the residual nucleus. They also stress that at $W \approx M\left(N^{*}\right)$ the data are above the results of the DWBA calculation, while at $W \approx M(\Delta)$ the trend is opposite. This indicates that FSI of $N^{*}$ and $\Delta$ are largely different, owing to their different quantum numbers.

In this note we propose a mechanism which is in the spirit of the suggestions of Ref. [1] and can account for an increase of $N^{*}$ production in nuclei, critically depending on $p_{m}$. We give numerical estimations of the effect and suggest some possible experimental developments.

Due to the nuclear Fermi motion, the kinematics of the $\gamma^{*} N$ hard collision is not fully determined, even when each component of the exchanged four momentum $q^{\mu}$ is known. Let $\Phi(k)$ be the amplitude of finding a bound nucleon with a momentum $k^{\mu} ; s=(q+k)^{2} \equiv M^{2}$ is the squared center of mass energy of the $\gamma^{*} N$ system, and the squared mass of the baryon state produced after the $\gamma^{*}$ absorption on the nucleon; $\Phi(k)$ induces an amplitude $f(M)$ for the values of this mass. So, according to the quantum superposition principle, many mass eigenstates are simultaneously produced in a $\gamma^{*} N$ collision in nucleus. Mass eigenstates diagonalize the free hamiltonian, but do not diagonalize the hamiltonian which describes the propagation of a hadronic wave packet inside a nucleus. In fact the interaction with the nuclear environment can convert a nucleon into a resonance and viceversa, or different resonances into each other. So the set of states produced in the 
initial hard collision is furtherly remixed by FSI (this mechanism is similar to the regeneration phenomenon in the $K^{o} \bar{K}^{o}$ system). In the nuclear $N^{*}$ electroproduction, different Feynman graphs sum coherently as shown in Fig. 1. In some kinematical regions the non-direct contribution can dominate the process. For example, if we measure $\left(e, e^{\prime} N^{*}\right)$ at the quasi-elastic peak of nucleon production, then the direct production of a $1535 \mathrm{MeV}$ mass in the $\gamma^{*} N$ collision is a very rare event, confined to the tail at large $|\vec{k}|$ of $\Phi(k)$. At the quasi-elastic peak, a much stronger possibility is given by a two-step process where a nucleon is produced in the initial hard scattering, and then converted by FSI into $N^{*}(1535)$. At other kinematics the easiest way to produce $N^{*}(1535)$ can be first to excite a higher mass state (e.g. $\left.S_{11}(1650)\right)$ and then to convert it into $N^{*}(1535)$ via FSI.

From now onwards the suffix $d$ represents the detected "particle" $X$ in $\left.\left(e, e^{\prime} X\right)\right)$. The missing momentum $\vec{p}_{m} \equiv \vec{p}_{d}-\vec{q}$ is a parameter of $\Phi(k)$, and consequently of $f(s)$ : for $\vec{k}$ equal to $p_{m}$ one has $s=(q+k)^{2}=M_{d}^{2}$. Every value of $\vec{k}$ different from $\vec{p}_{m}$ will lead to some event that will not be directly seen. While $\vec{p}_{m}$ is measurable, $\vec{k}$ is not measurable because it enters a loop (see Fig. 1).

If the detected particle is $N^{*}(1535)$, the curves in Fig. 2 represent the $\left(q, p_{m}\right)$ pairs that lead to the largest direct production of some states with given mass. Parallel kinematics $\left(\vec{p}_{m}\right.$ along $\left.\vec{q}\right)$ is assumed in all figures. Positive (negative) $p_{m}$ values correspond to $\vec{p}_{m}$ being parallel (antiparallel) to $\vec{q}$. The $q$-axis is the maximum for direct $N^{*}(1535)$ production. A DWBA calculation without final remixing shows a significant $N^{*}$ production only in a narrow band along the $q$-axis. But if we introduce the FSI remixing with 
the nucleon channel, then we can have a two-step $N^{*}$ production in the region of negative $p_{m}$, around the maximum for nucleon direct production. In the region of positive $p_{m}$ strong two-step contributions may come from the remixing with the resonances of the third region. Unfortunately there are many of them (see e.g. Ref. [2]) and their collective contribution is not easy to quantify if one does not know all the amplitudes by which they enter the process.

For each intermediate channel $|i\rangle$ leading to a given final state $|j\rangle$ we have to consider two amplitudes: $B_{i}$ for producing the intermediate state, and $f_{i j}$ associated with FSI ( $f_{i j}$ enter linearly into $V_{i j}$, see Ref. [3]). The relative phase and strength of the $i$-channel contribution to $|i\rangle$ production is given by $B_{i} \cdot f_{i j}$. In the regions where one channel (either direct or not) dominates, the results will not depend on the phase of $B_{i} \cdot f_{i j}$.

At not too low energies the wave function of a state with mass $M_{i}$ is approximately of the form $D(\vec{r}) \cdot \exp \left(i \vec{p}_{i} \cdot \vec{r}\right)$ (with $D(\vec{r})$ slowly varying with $\vec{r}$ ). For a given energy $E$, each $p_{i}$ is determined by $p_{i}=\sqrt{E^{2}-M_{i}^{2}}$. So the coherence between the wave functions in different channels is quickly lost if their masses are very different. For this reason the mixing of $N^{*}(1535)$ with a state, say, with $\mathrm{M}=3 \mathrm{GeV}$ is in practice much less important than the mixing with a state at $1.7 \mathrm{GeV}$.

We work in a time-independent coupled-channel DWBA formalism, with final state wave function formed by three coupled components, labelled 1,0 and 2, respectively: 1) direct $\left.N^{*}(1535), 0\right)$ nucleon, 2) a representative upper mass state $N^{*}(1680)$. These are simultaneously produced with amplitudes $B_{i}\left(Q^{2}, s\right)$ in the hard scattering at a given point $\vec{r}_{o}$. A common phase $\exp (i \vec{q}$. 
$\vec{r}_{o}$ ) is given by the virtual photon $\gamma^{*}$. Then we calculate a function of the form $\exp \left(i \vec{q} \cdot \vec{r}_{o}\right) \cdot \Sigma_{i} B_{i} \Psi_{i}\left(\vec{r}, \vec{r}_{o}\right)\left(\Psi_{i}\left(\vec{r}_{o}, \vec{r}_{o}\right) \equiv 1\right)$, which is an eigenfunction of the final state hamiltonian $H_{o}+V(3 \times 3$ matrix $)$. All the three channels describe particles propagating in the same direction with the same energy, and $p_{i}=$ $\sqrt{E^{2}-M_{i}^{2}} . V \equiv\left\{V_{i j}, i, j=0,1,2\right\}$ is an optical potential describing FSI. Its nondiagonal terms cause transitions between different channels. $V$ does not conserve flux $\left(V^{\dagger} \neq V\right)$, but each of its elements conserves energy. The total wave function $\Psi(\vec{r})$ is a coherent sum of all the waves of the above kind emitted from any nuclear point $\vec{r}_{o}$.

The details of the formalism applied to the coupled channel problem can be found in Ref. [3]. Some approximations have been removed since then (Ref. [4]). Numerical calculations have been carried out for $\left(e, e^{\prime} N^{*}\right)$ on ${ }^{27} A l$ with shells up to $l=2$ (harmonic oscillator wave functions). $\left|B_{i}\right|$ is calculated as in Ref. [4]:

$$
\left|B_{i}\right|^{2}=\int d^{3} k\left|g_{i}\left(Q^{2}\right)\right|^{2} R_{i}(s) n(k),
$$

where $s \equiv(q+k)^{\mu}(q+k)_{\mu}, n(k)$ is a Woods-Saxon distribution for the bound nucleon momentum $\vec{k}$,

$$
R_{i}(s)=\frac{M_{i} \Gamma_{i}}{\left(s-M_{i}^{2}\right)+M_{i}^{2} \Gamma_{i}^{2}} .
$$

The function $g_{i}\left(Q^{2}\right)$ is the vertex form factor. Since only the ratio $B_{i} / B_{j}$ is important here, all the $g\left(Q^{2}\right)$ are assumed equal. This is correct in the $Q^{2}$ range where the quark counting rule can be applied [5], and it is a good estimate at $Q^{2} \sim$ some $\mathrm{GeV}^{2}$. 
The amplitude $B_{i}$ is a function of $\vec{p}_{m} \equiv \vec{p}_{d}-\vec{q}$. Using four-momentum conservation in the hard vertex, and energy conservation in the rescattering (which is correct in the optical model), one can express $s$ (and so $\left|B_{i}\right|$ ) as a function of $\vec{q}$ and $\vec{p}_{m}$ :

$$
s-M_{i}^{2}=2 \vec{q} \cdot\left(\vec{p}_{m}-\vec{k}\right)+p_{m}^{2}-k^{2}+M_{d}^{2}-M_{i}^{2} .
$$

The behaviour of the maxima of $\left|B_{i}\right|$ is shown in Fig. 3. Since the relevant phase here is that of the product $B_{i} \cdot f_{i j}$, we absorb the phase of $B_{i}$ into $f_{i j}$.

All the nondiagonal FSI amplitudes $f_{i j}$ are chosen (in modulus) as $1 / 2$ the diagonal ones (all equal among them). This can be estimated from the diffraction resonance production data [6]. The phases are unknown to us. We have tested two opposite possibilities:

i) The relative phases of diagonal and nondiagonal terms are opposite (all equal inside each subgroup); this would lead to the largest nuclear transparency at $Q^{2} \rightarrow \infty$.

ii) All terms with the same phase (Fig. 4); this would lead to the smallest nuclear transparency at $Q^{2} \rightarrow \infty$.

The ratio $R$ between our results and PWBA is shown in Fig. 3 corresponding to the choice $i$ ) for the phases of the superposition amplitudes. The calculation for the choice $i i$ ) gives the same results for large and negative $p_{m}$, thus confirming that $N^{*}$ is mainly produced via nucleon convertion in FSI. This contribution is locally strong and independent of the above phases. For $p_{m} \gg 0$ we clearly identify the contribution by the upper resonance but, as 
noted above, we cannot say much about the role played by many coherent resonances. Anyway, unless many of them can sum up their contributions, we espect the interference with the nucleon to remain the most important. In parallel kinematics, this appears at large and negative $p_{m}$. Recalling eq. (3) we notice that, at the leading order in $p_{m} / q,\left|B_{i}\right|^{2}$ only depends on $\vec{p}_{m}$ via its component along $\vec{q}$, and the sign is crucial. So this effect should be more pronounced in the kinematics close to parallel.

There is a qualitative agreement between our results and the observations in Ref. [1]: an enhancement of $N^{*}$ production occurs at increasing absolute values of missing momentum. We stress however that the experiment of Ref. [1] did not distinguish between positive and negative missing momenta.

In this sense we suggest a measure of the asymmetry

$$
A \equiv \frac{\sigma\left(\vec{p}_{m} \cdot \hat{q}>p_{o}\right)-\sigma\left(\vec{p}_{m} \cdot \hat{q}<-p_{o}\right)}{\sigma\left(\vec{p}_{m} \cdot \hat{q}>p_{o}\right)+\sigma\left(\vec{p}_{m} \cdot \hat{q}<-p_{o}\right)},
$$

where a threshold $p_{o}$ is needed in order to exclude the central region where the direct $N^{*}$ production is dominant. This asymmetry is to large extent model independent and is equal to zero in PWBA. It should be very sensitive to the suggested interference effects.

We are grateful to L.B. Weinstein for useful discussions. 


\section{References}

[1] L.B. Weinstein et al., Phys. Rev. C 47, 225 (1993).

[2] P. Stoler, Phys. Rev. Lett. 66, 1003 (1991); Phys. Rev. C 44, 73 (1991); V. Burkert and Z. Li, Phys. Rev. D 47, 46 (1993).

[3] A. Bianconi, S. Boffi and D.E. Kharzeev, Phys. Lett. 305B, 1 (1993).

[4] A. Bianconi, S. Boffi and D.E. Kharzeev, Nucl. Phys. A, in press.

[5] V.A. Matveev, R.M. Muradyan and A.N. Tavkhelidze, Nuovo Cim. Lett. 7, 719 (1973); S.J. Brodsky and G.R. Farrar, Phys. Rev. Lett. 31, 1153 (1973).

[6] G. Alberi and G. Goggi, Phys. Rep. 74, 1 (1981); K. Goulianos, Phys. Rep. 101, 169 (1983). 


\section{Figure captions}

Fig. 1. The process of $N^{*}$ electroproduction off nucleus by hard scattering.

Fig. 2. The maxima of the squared effective amplitudes $\left|B_{2}\right|^{2}$ for the ${ }^{27} A l\left(e, e^{\prime} p\right)$ reaction as a function of four-momentum transfer $q(\mathrm{GeV})$ and missing momentum $p_{m}$ (in units of Fermi momentum $p_{F}=234 \mathrm{MeV}$ ). The curves are labelled according to the mass of the intermediate baryonic resonance mass.

Fig. 3. The transparency coefficient $R$ as a function of the momentum transfer $q$ (in $\mathrm{GeV}$ ) and the missing momentum $p_{m}$ (in units of the Fermi momentum $p_{f}=234 \mathrm{MeV}$ ). 